\title{
Botox hilft bei neuropathischem Schmerz
}

\author{
Zwei lokale Injektionen von Botulinumtoxin A lindern chronisch neuropathische, durch eine periphere \\ Nervenläsion bedingte Schmerzen besser als Placebo. Das zeigt die erste größere Studie zum Thema.
}

\begin{abstract}
Für eine doppelblinde, placebokontrollierte Studie wurden Patienten mit chronisch neuropathischen Schmerzen (mittlere Intensität $>4$ auf einer Skala von $0-10$, Dauer $\geq 6$ Monate) rekrutiert. Das Durchschnittsalter lag bei 52 Jahren. Die meisten hatten eine periphere Nervenschädigung. Zwei Drittel waren mit trizyklischen Antidepressiva vorbehandelt, 30\% mit Gabapentin oder Pregabalin, 50\% mit Opioiden. Sie erhielten im Abstand von zwölf Wochen entweder zwei s.c. Injektionen von bis zu 300 Einheiten Botulinumtoxin A im schmerzhaften Areal oder Placebo.

Bei den 34 Patienten der Botox-Gruppe lag die mittlere Schmerzintensität zu
\end{abstract}

Beginn bei 6,5. 24 Wochen nach der ersten Injektion war sie auf 4,6 gesunken. In der Placebogruppe ging der Wert signifikant schwächer von 6,4 auf 5,8 zurück. Der Therapieeffekt war bereits nach zwei Wochen nachweisbar.

Auch paroxysmale einschießende Schmerzen und Allodynie wurden unter Botulinumtoxin A signifikant reduziert. Die Werte auf einer Angstskala sowie die Schlafqualität verbesserten sich signifikant, nicht aber die Werte auf einer Depressionsskala.

\section{- Attal N, de Andrade DC, Adam F et al. Safety and efficacy of repeated injections of botulinum toxin $A$ in peripheral neuro- pathic pain (BOTNEP): a randomised, double-blind, placebo-} controlled trial. Lancet Neurol. 2016;15:555-65

\section{KOMMENTAR}

Man muss betonen, dass die Patienten bereits seit Jahren unter den Schmerzen litten und eine medikamentöse Therapie nicht ausreichend wirksam war. Der groBe Vorteil von Botulinumtoxin A ist, dass keine zentralen Nebenwirkungen auftreten. Die Autoren geben die Number needed to treat mit 7,3 an, was ungefähr im Bereich von Pregabalin läge. Derzeit ist die Botox-Behandlung noch Off-LabelUse. Sie ist aber erwägenswert, wenn andere Medikamente wegen Nebenwirkungen nicht toleriert werden, oder wenn Opioide wegen einer hohen Suchtgefährdung ausscheiden.

Prof. Dr. med. H.-C. Diener

\section{Nach der Koronarangiografie hatte er "so einen Hals"}
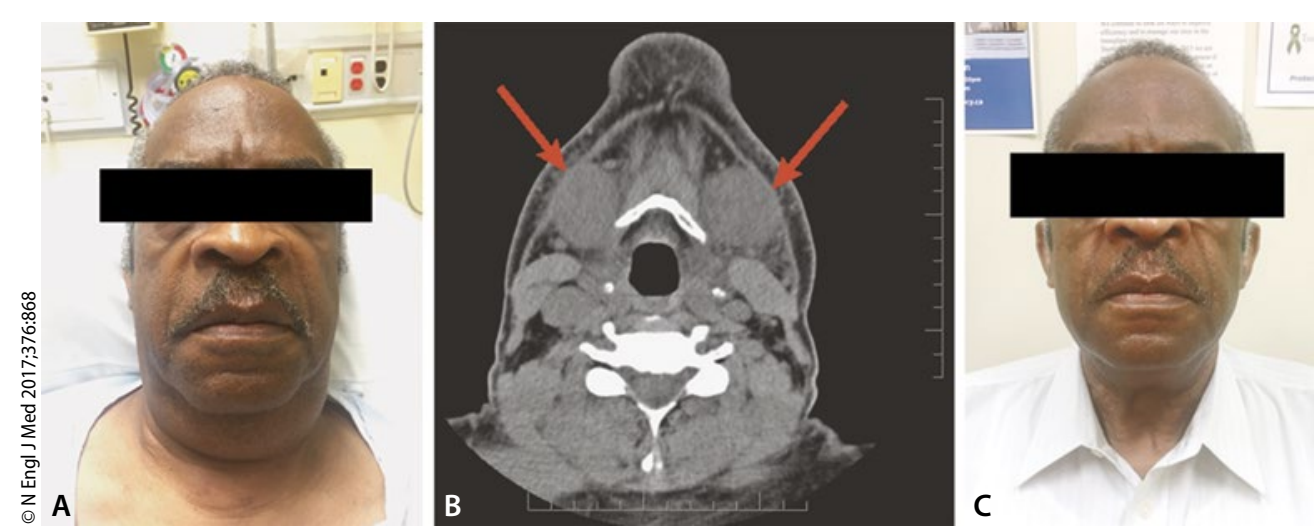

A: Schwellung am Hals. B: Symmetrisch vergrößerte Submandibulardrüsen im CT (Pfeile). C: Verbesserter Zustand nach fünf Tagen.

Ein 67-jähriger Mann stellte sich wegen einer Schwellung im Halsbereich, Dysphagie und Heiserkeit in der Nothilfe vor. Die Beschwerden waren einige Stunden nach einer Koronarangiografie aufgetreten. Der Patient hatte nach einer Herztransplantation eine chronische Nierenerkrankung entwickelt. Wegen einer bekannten Kontrastmittelallergie waren ihm vor der Koronarangiografie Hydrocortison und Diphenhydramin verabreicht worden. Palpato- risch waren beide Glandulae submandibulares vergrößert und druckdolent (Abb. A). Im CT von Kopf und Nacken erkannte man symmetrisch vergrößerte Submandibulardrüsen ohne Hinweise auf Steine (Abb. B).

Es handelt sich um eine jodassoziierte Sialoadenitis, die wahrscheinlich durch hohe Jodkonzentrationen im Speichel hervorgerufen wird. Die Ausführungsgänge schwellen an und behindern den Abfluss des Speichels. Bei Patienten mit Niereninsuffizienz ist dieses Risiko wegen der verzögerten Kontrastmittelausscheidung wohl erhöht. Die Beschwerden bilden sich in der Regel innerhalb einiger Tage auch ohne Behandlung zurück, bei diesem Mann dauerte es fünf Tage (Abb. C). Da es sich nicht um eine allergische Reaktion handelt, kann sie auch nicht durch eine entsprechende Prämedikation verhindert werden.

Prof. Dr. med. H. S. FüeßI

- Afshar M, Alhussein M (m.asfhar.h@gmail.com): lodine-associated sialadenitis. N Engl J Med 2017;376:868 